\title{
Collagen Tissue Engineering: Development of Novel Biomaterials and Applications
}

\author{
LIAN CEN, WEI LIU, LEI CUI, WENJIE ZHANG, AND YILIN CAO
}

\begin{abstract}
Department of Plastic and Reconstructive Surgery [W.L., L.C., W.Z., Y.C.], Shanghai 9th People's Hospital, Shanghai Jiao Tong University School of Medicine, Shanghai 200011, China; National Tissue Engineering Center of China [L.C., W.L., L.C., W.Z., Y.C.], Shanghai 200235, China
\end{abstract}

\begin{abstract}
Scientific investigations involving collagen have inspired tissue engineering and design of biomaterials since collagen fibrils and their networks primarily regulate and define most tissues. The collagen networks form a highly organized, three-dimensional architecture to entrap other ingredients. Biomaterials are expected to function as cell scaffolds to replace native collagen-based extracellular matrix. The composition and properties of biomaterials used as scaffold for tissue engineering significantly affect the regeneration of neo-tissues and influence the conditions of collagen engineering. The complex scenario of collagen characteristics, types, fibril arrangement, and collagen structure-related functions (in a variety of connective tissues including bone, cartilage, tendon, skin and cornea) are addressed in this review. Discussion will focus on nanofibrillar assemblies and artificial synthetic peptides that mimic either the fibrillar structure or the elemental components of type I collagen as illustrated by their preliminary applications in tissue engineering. Conventional biomaterials used as scaffolds in engineering collagencontaining tissues are also discussed. The design of novel biomaterials and application of conventional biomaterials will facilitate development of additional novel tissue engineering bioproducts by refining the currently available techniques. The field of tissue engineering will ultimately be advanced by increasing control of collagen in native tissue and by continual manipulation of biomaterials. (Pediatr
\end{abstract} Res 63: 492-496, 2008)

\section{GENERAL DESCRIPTION ON COLLAGEN IN CONNECTIVE TISSUES}

The extracellular matrix (ECM), provides physical support to tissues by occupying the intercellular space, acting not only as benign native scaffolding for arranging cells within connective tissues, but also as a dynamic, mobile, and flexible substance defining cellular behaviors and tissue function (1). For most soft and hard connective tissues (bone, cartilage, tendon, cornea, blood vessels, and skin) collagen fibrils and their networks function as ECM, the highly organized, three-dimensional (3D) architecture surrounding various cells. Collagen plays a dominant role in maintaining the biologic and structural integrity of ECM and is highly dynamic, undergoing constant remodeling for proper physiologic functions (1). Hence, the ideal goal of tissue

Received October 31, 2007; accepted January 4, 2008.

Correspondence: Yilin Cao, M.D., Ph.D., Department of Plastic and Reconstructive Surgery, Shanghai 9th People's Hospital, Shanghai Jiao Tong University School of Medicine, 639 Zhi Zao Ju Road, Shanghai, 200011, P. R. China; e-mail: yilincao@yahoo.com

This study is financially supported by National "973" (2005CB522700), and Shanghai $\mathrm{Pu}$ Jiang Foundation of Shanghai Science and Technology Development Committee (07pj14110, to L.C.). regeneration is to restore both the structural integrity and the vivid remodeling process of native ECM, especially restoring the delicate collagen networks under which normal physiologic regeneration occurs.

Collagen molecules have a triple-helical structure and the presence of 4-hydroxyproline resulting from a posttranslational modification of peptide-bound prolyl residues provides a distinctive marker of these molecules (2). To date, 28 collagen types have been identified; I, II, III, and V are the main types that make up the essential part of collagen in bone, cartilage, tendon, skin, and muscle. They also exist in fibrillar forms with elaborate 3D arrays in ECM (3-5).

Bone tissues are mainly constructed from type I with a small quantity of type $\mathrm{V}$ collagen forming a framework that anchors nanosized hydroxyapatite (HA) crystals. Both organic and mineral phases compose a closely interwoven and highly complex but ordered composite which is further organized into layers, or lamellae, that are a few microns thick. The lamellae are arranged into higher order structures. The organic framework is mainly constructed by type I collagen fibrils in elaborate 3D arrays as concentric weaves (4), and the anchored bone crystals are $50 \mathrm{~nm}$ long, $28 \mathrm{~nm}$ wide, and $2 \mathrm{~nm}$ thick (6).

Bioengineered bone was formed by collagen synthesis, fibril formation, assembling and crosslinking with mineralization by Ca-P crystals to form a continuous network and further remodeling. Collagen fibers, while providing the framework for HA deposits, are the principal source of tensile strength of bone tissues. The mechanical properties of bone can be adjusted by varying fibril orientation which may result from the degree of collagen crosslinking and the mineral-organic composite remodeling process $(7,8)$.

There are three main types of cartilage: hyaline articular cartilage, fibrocartilage, and elastic cartilage. This review will only discuss hyaline articular cartilage which is found on the surface of long bones in articulating joints and is extremely important in the lubrication and load distribution in the joint. Usually, articular cartilage is subdivided into three zones: superficial, middle, and deep zones (9).

Type II collagen is the chief component in articular cartilage and is approximately $60 \%$ of the dry weight of the tissue.

\footnotetext{
Abbreviations: DBM, demineralized bone matrix; ECM, extracellular matrix; GAG, glycosaminoglycans; HA, hydroxyapatite; PA, peptide-amphiphile; PGA, polyglycolide
} 
According to the work by Muir et al., small fibers with a diameter of less than $10 \mathrm{~nm}$ are distributed in all of its three zones (10). There are also large fibers of approximately $34 \mathrm{~nm}$ in diameter, oriented parallel to the surface in the superficial zone; fibers of 70-100 nm mostly arranged randomly in the middle zone; fibers of $\sim 140 \mathrm{~nm}$ organized radially in the deep zone. Trace amounts of types I, VI, IX, X, and XI collagens can also be detected (11).

Collagen fibers, together with $10 \%$ glycosaminoglycans (GAGs), contribute to the viscoelasticity and compressive function of articular cartilage, although the three zones differ in their viscoelastic and compressive properties. Assuming that different aligning patterns and distribution of collagen fibers account for this difference, cartilage engineering relies on successful engineering of collagen frameworks in different zones for further deposition of other proteins, especially proteoglycans or GAGs.

Tendon consists of type I fibrillar collagen along with trace quantities of types II, III, IV, V, and VI and is $60-85 \%$ collagen by dry weight. Tendon transmits the force of muscular contraction to bone $(9,12)$ and most tendon types experience tension, such as Achilles or patellar tendon, although a few types also experience a certain degree of compression, such as the supraspinatus tendon (13). Collagen fibrils in tendon vary in diameter from $30 \mathrm{~nm}$ to $300 \mathrm{~nm}$, depending on the stage of development (14). Usually a nonuniform or bimodal distribution of fibril diameters can be observed, which allows for tight lateral packing (15). These fibrils are generally oriented longitudinally to support the great tensile stress exerted on the tissue although some may be transversely arranged $(9,16)$. Collagen fibrils are essential in tendon for maintaining mechanical properties and for transmitting force during movements (5).

Skin is the body's largest organ composed primarily of epidermis, dermis, and hypodermis layers with a complex nerve and blood supply. Dermis constitutes the bulk of the skin and is composed of collagen with some elastin and GAGs cushioning the body against mechanical injury by conferring elasticity and plasticity to the skin (17). Seventy to $80 \%$ of the dry weight of the dermis is mainly type I collagen which exists in the form of loosely interwoven, large, wavy, randomly oriented bundles and within each bundle the collagen is packed together closely (18). Collagen types III and V have also been detected in the dermis of skin (5) and are primarily responsible for tensile strength. The dermal layer network also maintains stability of the epidermis (18).

The cornea consists of three distinct cellular layers: outer epithelium, central stroma, and inner endothelium. The central stroma occupies approximately $90 \%$ of the corneal volume and $70 \%$ of its dry weight and is composed of a dense and highly organized ECM of collagen fibrils and proteoglycans (19). The stroma is the major component of transparent corneal tissue and is mainly comprised of type I and V collagens existing in parallel-organized fibrils with a uniform diameter of around $30 \mathrm{~nm}$ (20) that are further arranged in lamellae. Intensive intra- and intermolecular covalent crosslinking takes place universally to stabilize the collagen fibrils against proteolytic degradation and the fibrils confer the desired tensile strength to the stroma. The 3D architecture of the thin collagen fibrils may contribute to cornea transparency (21).

The complex structure of fibrillar type I collagen presents different morphologies in different tissues performing different functions. When associated with HA crystals in bone, it provides rigid and shock-resistant tissues with high Young modulus. It can behave like an elastomer with low rigidity and high deformation to rupture in tendon. In cornea, it shows optical properties such as transparency. Capturing the intrinsic building blocks and more complex, higher order supramolecular structures of collagen has been the current focus of the biomaterial society.

\section{DEVELOPMENT OF NOVEL BIOMATERIALS}

In view of the variety of roles played by collagen in different tissues, research has focused on developing novel biomaterials to mimic the intricate fibrillar architecture which would be able to function as cell scaffolding replacing native collagen-based ECM.

One promising candidate is peptide-amphiphile (PA) nanofibers which are produced by molecular self-assembly as developed by Stupp and coworkers $(22,23)$. The selfassembling unit is a PA, a hydrophilic 11-residue peptide with an N-terminal alkyl tail of 16 carbon atoms. PAs are synthesized by standard solid phase chemistry that ends with the alkylation of the $\mathrm{NH}_{2}$ terminus of the peptide and they further self-associate to form cylindrical micelles or nanofibers because of the amphiphile's overall conical shape. The alkyl tails are packed in the centers of the fibers, while the peptide segments are exposed to the aqueous environment so the chemistry of the peptide region is repetitively displayed on their surface. The incorporation of four consecutive cysteine amino acids helps to stabilize the fibrous supramolecular structure and the introduction of a phosphoserine residue allows the fiber to display a highly phosphorylated surface to facilitate the formation of calcium phosphate minerals. The sequence of Arg-Gly-Asp in the peptide is expected to promote the adhesion and growth of cells on fiber surfaces. The diameter of yielded PA fibers is $\sim 7 \mathrm{~nm}$ and the length could be up to several micrometers. Mineralization of the fibers was demonstrated and showed that plate-shaped polycrystalline minerals deposited on the PA fibers were consistent with HA found in native bone tissue. Thus, these mineralized nanofibers resemble the lowest level of hierarchical organization of bone $(22,23)$. Furthermore, the same group extended their work in such supramolecular structures by synthesizing selfassembling oligomeric-amphiphiles that allow incorporation of specific biomolecular signals, which is expected to direct and regulate the cellular behaviors (24).

Zhang developed a class of nanofibrillar gels crosslinked by self-assembly of self-complementary amphiphilic peptides in physiologic medium (25). The resulting gels have more than 90\% water content and the formed matrices are made of interwoven nanofibers with diameters of $\sim 10 \mathrm{~nm}$ and pores of $\sim 200 \mathrm{~nm}$ (26). These simple, defined, and tailor-made selfassembling peptides have provided de novo-designed scaffolds for $3 \mathrm{D}$ cell culture and they biomechanically organize cells in 
a 3D fashion. Continued efforts to control nanofibrillar morphologies include the elucidation of rational design principles to better control the production of kinked, wavy, or branched fibers (27).

The development of artificial collagen-like materials is another important area of research. By using native chemical ligation techniques that synthetic peptide units spontaneously polymerize in aqueous solutions, Paramonov et al. prepared poly(Xaa-Yaa-Gly $)_{n}$ which are collagenous peptide polymers with an apparent molecular weight less than or equal to 1,000,000 D (28). The triple-helical polymers can also form a nanofiber-like structure with a length in micrometers. Since the polymerization proceeds under mild conditions, it is possible to incorporate amino acid residues with various sidechain functional groups. The presence of Cys residues on the polymers also enables further crosslinking and modification by functional moieties. However, only limited chain length can be achieved. Koide et al. (29), Kotch and Raine (30) also independently developed a novel peptide-based system for obtaining collagen-like triple-helical supramolecules via a spontaneous self-assembly process. Their peptide units are self-complementary trimers based on Pro-Hyp-Gly repeats. Peptide strands are tethered together by two disulphide bridges in a staggered arrangement in the trimer. Their design allows the intermolecular folding to form elongated triplehelical supramolecules $(29,30)$.

Although the nanostructures described above are oversimplified mimics of natural ECM and lack essential natural temporal and spatial complexity, they do represent advances in materials engineering and cell biology. It is expected that the increasing symbiosis would ultimately give out synthetic materials that possess both the necessary signals to recapitulate developmental processes in tissue regeneration. The ideal artificial collagen-mimetic biomaterials should mimic both the physical and biochemical properties of native collagens. However, the supramolecular structure significantly contributes to the physical properties of those native fibrillar collagens when acting as biomaterials. Thus, for the current collagen-mimic nanofibers or synthetic polymers to be further applied as biomaterials in modern medical fields, especially in tissue engineering, efforts are still needed to gain more control over the high order structures of native collagens.

\section{APPLICATION OF BIOMATERIALS}

Biomaterials should include all materials or systems proposed for clinical applications to replace part of a living system or to function in intimate contact with living tissues. The leading technology of tissue engineering is one of the most challenging and important applications of biomaterials, as it based on a 3D bioartificial scaffold (31). The formation of desired neotissues is expected together with the gradual degradation of the scaffold while the collagen engineering during tissue formation is the intrinsic target to be achieved. However, it is highly dependent on the acceptance of the artificial implant by the surrounding tissues and on the elicited specific cellular responses as well as on the biomechanical, structural, and degradation properties of the biomaterials (32). As to those novel biomaterials addressed in the previous section, only preliminary investigations on their application in tissue engineering were carried out, while most studies are still in the stage of in vitro cellular compatibility.

The PA nanofibers developed by Stupp and coworkers were applied to prepare a hybrid bone implant material (33) and PA nanofibers were combined with a $\mathrm{Ti}-6 \mathrm{Al}-4 \mathrm{~V}$ foam to do so. The PA molecules self-assembled into a nanofiber matrix within the pores of the metallic foam, fully occupying the foam's interconnected porosity. The hybrid matrix allows the encapsulation of cells, whereas the nanofibers nucleate mineralization of calcium phosphate phases with a $\mathrm{Ca}: \mathrm{P}$ ratio consistent with that of HA. When the hybrid material was used to repair a bone defect (a hole $2 \mathrm{~mm}$ in diameter) in the hind femur of a rat, collagenous fiber formation and early deposition of bone adjacent to the implant exterior was observed (33). However, further quantitative and statistical analysis of the in vivo bone formation conditions as well as the long-term responses remain to be addressed.

The nanofibrillar gels developed by Zhang and coworkers demonstrated support of neuronal cell attachment and differentiation as well as extensive neurite outgrowth (34). Those gels maintained functions of differentiated chondrocytes and promoted differentiation of liver progenitor cells $(35,36)$. Furthermore, they could also be used for brain repair (37). These preliminary results substantiate the potential of nanofibrillar gels as biomaterials but applications for those designed via biomimic techniques are still in development and the ability to engineer collagen tissues still needs exploration.

In contrast, conventional biomaterials have been successfully applied to tissue engineering. These biomaterials are not so delicate and specific in their design; their structure and function differ significantly from the collagen-based ECM. There is a lot of convincing in vivo data demonstrating their applications in engineering collagen tissues including bone, cartilage, skin, tendon, cornea, etc.

Animal-derived and recombinant collagens, especially type I, are acknowledged as one of the most useful biomaterials available and are now widely used for tissue engineering, cosmetic surgery, and drug delivery systems. They are used either in their native fibrillar form or after denaturation in variously fabricated forms, such as sponges, sheets, plugs, and pellets. Butler and coworkers used type I collagen sponges to engineer patellar tendons in rabbits under different culture conditions (38-40). Using the collagen sponge combined with bone marrow-derived mesenchymal stem cells, they demonstrated that the engineered neotissue almost reached about three-fourth of the mechanical properties of normal tissue (39). Enhancement of collagen type I and type III expression by seeding cells was confirmed by gene-level assays (40). Bovine collagen type I was proposed and evaluated as 3D scaffolds for reestablishing the collagen fibrillar structure of the skin. Such skin substitutes based on cellseeded collagens have been commercialized extensively (e.g., Apligraf from Organogenesis, Inc. and OrCel from Ortec Int.). The feasibility of using collagen as biomaterials for engineering collagen (type I) tissues in tendon and skin can be ascertained but the use of these animal-derived collagens in human 
is sometimes compromised by possible allergic reactions and pathogen transmission and the use of recombinant collagens may suffer from the lack of biologic activities of native tissue. This is because recombinant collagen does not undergo significant posttranslational modifications (41).

Synthetic polymers are alternatively favored conventional biomaterials because they can be manufactured from a wide range of biodegradable polymers with easy processability, controlled degradation, and susceptibility to modification $(42,43)$ which makes custom design possible. This sub-group of hydrolytically degradable polymers has gained increasing acceptance, including $\operatorname{poly}(\alpha$-esters), polyurethanes, poly(ester amide), poly(ortho esters), polyanhydrides, poly(propylene fumarate) and so on. $\operatorname{Poly}(\alpha$-hydroxy acid)s in the class of poly( $\alpha$-esters), such as polyglycolide (PGA), is the one that gained extensive attention and has the widest applicability. Scaffolds made from PGA fibers have been thoroughly investigated.

PGA fibers when combined with tenocytes, or dermal fibroblasts, were successfully applied to engineer a flexor digitorum profundus tendon and a flexor digital superficial tendon in animal models, respectively $(44,45)$. The synthesis of type I collagen fibrils and further remodeling by seeded cells were confirmed. Furthermore, scaffolds of the same PGA fibers were used to engineer a cornea stroma of a rabbit (46). The construct of PGA/cells gradually became transparent over time and the generated collagen fibrils were spatially arranged in a highly organized configuration parallel with the corneal surface. The diameter of collagen fibrils and their distribution were also similar to native fibrils. Although further remodeling of the formed collagen fibrils either in engineered tendons or cornea stroma is still required to match native tissue, the possibility of using PGA fibers to engineer type I collagen tissues in tendon and in cornea was demonstrated.

The same PGA fibers, when arranged into 3D templates, were combined with either chondrocytes or bone marrow stromal cells to repair large full-thickness defects of articular cartilage in either weight-bearing areas or nonweight bearing areas $(47,48)$. Expression of collagen II was enhanced at both protein and gene levels of the seeding cells and the defects were repaired by engineered hyaline cartilage with a smooth articular surface indistinguishable from nearby normal tissue.

Engineering type II collagen tissues in cartilage, PGA fibers could be viable scaffolds. However, the application of PGA suffers from acidic degradation products which would worsen the $\mathrm{pH}$ environment surrounding the cells resulting in unfavorable host responses.

The last area of this focus is on inorganic materials, such as coral, calcium alginate, and demineralized bone matrix (DBM). Those materials are relatively simple to process compared with synthetic polymers, and their main application is hard connective tissues, such as bone. Although their microstructure is not designed artificially to simulate collagen-based ECM, especially coral and DBM, they have been successfully used to repair bone tissues.

DBM and coral can be used to repair nonweight-bearing zones of bone such as cranial bone defects where bone formation in the defect site has been achieved (49,50). Coral can also be used to repair weight bearing bones such as femoral bone defects and complete bone union healing has also been achieved (51). When injectable bone was required for surgical convenience, calcium alginate could be a better choice as it has been used to repair sheep cranial defects and alveolar bone defects, respectively $(52,53)$.

No direct qualitative and quantitative assays were performed to envisage type I collagen formation in engineered bones and the only indication of success was by comparing the similarity of the microstructures between engineered and natural bone. Quality control is difficult and the application of this biomaterial has disadvantages such as nonuniform porosity which affects its mechanical properties.

Development of novel biomaterials that overcome these intrinsic drawbacks is necessary. Techniques and experiences on how to process those conventional materials for acting as suitable scaffolds and to contruct the scaffold/cell composite as well as the in vivo surgical procedures are valuable and continue to advance. These advancements will further guide and facilitate expansion of applications for novel biomaterials.

\section{CONCLUDING REMARKS}

Collagen, as the basic structural element for most connective tissues, plays a prominent role in maintaining the biologic and structural integrity of ECM architecture and presents different morphologies in different tissues which perform different functions. This versatility inspired biomaterial researchers to develop novel biomaterials resembling both structures and bioactivities of the collagens, especially type I fibrillar collagen. Mimicking the nanoscaled structures and the molecular components of native collagen fibrils shed light on the desired bioactivities of those novel biomaterials. Although only oversimplified mimics can be reached currently, they do represent advances in materials engineering and cell biology. However, current application is still in its infancy.

Conventional biomaterials are designed and fabricated easily and have already found applications in engineering collagen tissues. Using biomaterials such as animal-derived collagen and PGA during the engineering process provided invaluable experiences in such areas as scaffold fabrication, scaffold/cell composite construction, surgical operation and sampling, and development of histologic and biologic evaluation assays on the neotissues. The application of these biomaterials has provided further insight into biomaterial-cellular interactions. Further refinement of current biotechnology will enhance the evolution of tissue engineering in the future. To promote further development of tissue engineering and clinical applicability of these products, it is important to optimize fabrication and application that provides satisfactory interaction and imitation of biologic functions. Multidisciplinary research will produce synthetic materials that will enable cells to exist in a benign environment and restore collagen engineering production that is similar to the natural developmental processes, or it will create an environment with signals that simulate developmental processes of the human body in tissue regeneration. 


\section{REFERENCES}

1. Aszódi A, Legate KR, Nakchbandi I, Fässler R 2006 What mouse mutants teach us about extracellular matrix function. Annu Rev Cell Dev Biol 22:591-621

2. Grant ME 2007 From collagen chemistry towards cell therapy-a personal journey. Int J Exp Pathol 88:203-214

3. Veit G, Kobbe B, Keene DR, Paulsson M, Koch M, Wagener R 2006 Collagen XXVIII, a novel von Willebrand factor A domain-containing protein with many imperfections in the collagenous domain. J Biol Chem 281:3494-3504

4. Canty EG, Kadler KE 2005 Procollagen trafficking, processing and fibrillogenesis. J Cell Sci 118:1341-1353

5. Smith K, Rennie MJ 2007 New approaches and recent results concerning humantissue collagen synthesis. Curr Opin Clin Nutr Metab Care 10:582-590

6. Cui FZ, Li Y, Ge J 2007 Self-assembly of mineralized collagen composites. Mater Sci Eng R Rep 57:1-27

7. Taylor D 2007 Fracture and repair of bone: a multiscale problem. J Mater Sci 42:8911-8918

8. Buehler MJ, Ackbarow T 2007 Fracture mechanics of protein materials. Mater Today 10:46-58

9. Johns DE, Athanasiou KA 2007 Design characteristics for temporomandibular joint disc tissue engineering: learning from tendon and articular cartilage. Proc Inst Mech Eng [H] 221:509-526

10. Muir H, Bullough P, Maroudas A 1970 The distribution of collagen in human articular cartilage with some of its physiological implications. J Bone Joint Surg Br 52:554-563

11. Naumann A, Dennis JE, Awadallah A, Carrino DA, Mansour JM, Kastenbauer E, Caplan AI 2002 Immunochemical and mechanical characterization of cartilage subtypes in rabbit. J Histochem Cytochem 50:1049-1058

12. Vogel KG, Peters JA 2005 Histochemistry defines a proteoglycan-rich layer in bovine flexor tendon subjected to bending. J Musculoskelet Neuronal Interact 5:64-69

13. Fan L, Sarkar K, Franks DJ, Uhthoff HK 1997 Estimation of total collagen and types I and III collagen in canine rotator cuff tendons. Calcif Tissue Int 61:223-229

14. Fleischmajer R, Perlish JS, Timpl R, Olsen BR 1988 Procollagen intermediates during tendon fibrillogenesis. J Histochem Cytochem 36:1425-1432

15. Ottani V, Raspanti M, Ruggeri A 2001 Collagen structure and functional implications. Micron 32:251-260

16. Evanko SP, Vogel KG 1990 Ultrastructure and proteoglycan composition in the developing fibrocartilaginous region of bovine tendon. Matrix 10:420-436

17. Metcalfe AD, Ferguson MW 2007 Tissue engineering of replacement skin: the crossroads of biomaterials, wound healing, embryonic development, stem cells and regeneration. J R Soc Interface 4:413-437

18. Waller JM, Maibach HI 2006 Age and skin structure and function, a quantitative approach (II): protein, glycosaminoglycan, water, and lipid content and structure. Skin Res Technol 12:145-154

19. Torbet J, Malbouyres M, Builles N, Justin V, Roulet M, Damour O, Oldberg A, Ruggiero F, Hulmes DJ 2007 Orthogonal scaffold of magnetically aligned collagen lamellae for corneal stroma reconstruction. Biomaterials 28:4268-4276

20. Hirsch M, Prenant G, Renard G 2001 Three-dimensional supramolecular organization of the extracellular matrix in human and rabbit corneal stroma, as revealed by ultrarapid-freezing and deep-etching methods. Exp Eye Res 72:123-135

21. Meek KM, Leonard DW, Connon CJ, Dennis S, Khan S 2003 Transparency, swelling and scarring in the corneal stroma. Eye 17:927-936

22. Hartgerink JD, Beniash E, Stupp SI 2001 Self-assembly and mineralization of peptide-amphiphile nanofibers. Science 294:1684-1688

23. Hartgerink JD, Beniash E, Stupp SI 2002 Peptide-amphiphile nanofibers: a versatile scaffold for the preparation of self-assembling materials. Proc Natl Acad Sci USA 99:5133-5138

24. Niece KL, Hartgerink JD, Donners JJ, Stupp SI 2003 Self-assembly combining two bioactive peptide-amphiphile molecules into nanofibers by electrostatic attraction. J Am Chem Soc 125:7146-7147

25. Zhang S 2003 Fabrication of novel biomaterials through molecular self-assembly. Nat Biotechnol 21:1171-1178

26. Marini DM, Hwang W, Lauffenburger DA, Zhang S, Kamm RD 2002 Left-handed helical ribbon intermediates in the self-assembly of a $\beta$-sheet peptide. Nano Lett 2:295-299

27. Ryadnov MG, Woolfson DN 2003 Engineering the morphology of a self-assembling protein fibre. Nat Mater 2:329-332

28. Paramonov SE, Gauba V, Hartgerink JD 2005 Synthesis of collagen-like peptide polymers by native chemical ligation. Macromolecules $38: 7555-7561$
29. Koide T, Homma DL, Asada S, Kitagawa K 2005 Self-complementary peptides for the formation of collagen-like triple helical supramolecules. Bioorg Med Chem Lett 15:5230-5233

30. Kotch FW, Raines RT 2006 Self-assembly of synthetic collagen triple helices. Proc Natl Acad Sci USA 103:3028-3033

31. Langer R, Vacanti JP 1993 Tissue engineering. Science 260:920-926

32. Sanchez C, Arribart H, Guille MM 2005 Biomimetism and bioinspiration as tools for the design of innovative materials and systems. Nat Mater 4:277-288

33. Sargeant TD, Guler MO, Oppenheimer SM, Mata A, Satcher RL, Dunand DC, Stupp SI 2008 Hybrid bone implants: self-assembly of peptide amphiphile nanofibers within porous titanium. Biomaterials 29:161-171

34. Holmes TC, de Lacalle S, Su X, Liu G, Rich A, Zhang S 2000 Extensive neurite outgrowth and active synapse formation on self-assembling peptide scaffolds. Proc Natl Acad Sci USA 97:6728-6733

35. Kisiday J, Jin M, Kurz B, Hung H, Semino C, Zhang S, Grodzinsky AJ 2002 Self-assembling peptide hydrogel fosters chondrocyte extracellular matrix production and cell division: implications for cartilage tissue repair. Proc Natl Acad Sci USA 99:9996-10001

36. Semino CE, Merok JR, Crane GG, Panagiotakos G, Zhang S 2003 Functional differentiation of hepatocyte-like spheroid structures from putative liver progenitor cells in three-dimensional peptide scaffolds. Differentiation 71:262-270

37. Ellis-Behnke RG, Liang YX, You SW, Tay DK, Zhang S, So KF, Schneider GE 2006 Nano neuro knitting: peptide nanofiber scaffold for brain repair and axon regeneration with functional return of vision. Proc Natl Acad Sci USA 103:50545059

38. Awad HA, Boivin GP, Dressler MR, Smith FN, Young RG, Butler DL 2003 Repair of patellar tendon injuries using a cell-collagen composite. J Orthop Res 21:420431

39. Juncosa-Melvin N, Shearn JT, Boivin GP, Gooch C, Galloway MT, West JR, Nirmalanandhan VS, Bradica G, Butler DL 2006 Effects of mechanical stimulation on the biomechanics and histology of stem cell-collagen sponge constructs for rabbit patellar tendon repair. Tissue Eng 12:2291-2300

40. Juncosa-Melvin N, Matlin KS, Holdcraft RW, Nirmalanandhan VS, Butler DL 2007 Mechanical stimulation increases collagen type I and collagen type III gene expression of stem cell-collagen sponge constructs for patellar tendon repair. Tissue Eng 13:1219-1226

41. Koide T 2007 Designed triple-helical peptides as tools for collagen biochemistry and matrix engineering. Philos Trans R Soc Lond B Biol Sci 362:1281-1291

42. Chung HJ, Park TG 2007 Surface engineered and drug releasing pre-fabricated scaffolds for tissue engineering. Adv Drug Deliv Rev 59:249-262

43. Nair LS, Laurencin CT 2007 Biodegradable polymers as biomaterials. Prog Polym Sci 32:762-798

44. Cao Y, Liu Y, Liu W, Shan Q, Buonocore SD, Cui L 2002 Bridging tendon defects using autologous tenocyte engineered tendon in a hen model. Plast Reconstr Surg 110:1280-1289

45. Liu W, Chen B, Deng D, Xu F, Cui L, Cao Y 2006 Repair of tendon defect with dermal fibroblast engineered tendon in a porcine model. Tissue Eng 12:775-788

46. Hu X, Liu W, Cui L, Wang M, Cao Y 2005 Tissue engineering of nearly transparent corneal stroma. Tissue Eng 11:1710-1717

47. Liu Y, Chen F, Liu W, Cui L, Shang Q, Xia W, Wang J, Cui Y, Yang G, Liu D, Wu J, Xu R, Buonocore SD, Cao Y 2002 Repairing large porcine full-thickness defects of articular cartilage using autologous chondrocyte-engineered cartilage. Tissue Eng 8:709-721

48. Zhou G, Liu W, Cui L, Wang X, Liu T, Cao Y 2006 Repair of porcine articular osteochondral defects in non-weight bearing areas with autologous bone marrow stromal cells. Tissue Eng 12:3209-3221

49. Liu W, Cui L, Cao Y 2006 Bone reconstruction with BMSC. Methods Enzymol 420:362-380

50. Cui L, Liu B, Liu G, Zhang W, Cen L, Sun J, Yin S, Liu W, Cao Y 2007 Repair of cranial bone defects with adipose derived stem cells and coral scaffold in a canine model. Biomaterials 28:5477-5486

51. Zhu L, Liu W, Cui L, Cao Y 2006 Tissue-engineered bone repair of goat-femur defects with osteogenically induced bone marrow stromal cells. Tissue Eng 12:423 433

52. Shang Q, Wang Z, Liu W, Shi Y, Cui L, Cao Y 2001 Tissue-engineered bone repair of sheep cranial defects with autologous bone marrow stromal cells. J Craniofac Surg $12: 586-593$

53. Weng Y, Wang M, Liu W, Hu X, Chai G, Yan Q, Zhu L, Cui L, Cao Y 2006 Repair of experimental alveolar bone defects by tissue engineered bone. Tissue Eng $12: 1503-1513$ 